\title{
Study on the Application of Neural Network in the Computer Network Security Evaluation
}

Xue-rui WANG, Yan ZHOU

Henan Institute of Engineering

Zhengzhou, Zip Code: 450007

China

ABSTRACT: Research computer network security problem. There were nonlinear relations among the evaluation inde xes, and it was difficult for an accurate mathematical model to describe the nonlinear relationship. In order to improve the evaluation accuracy of computer network security, this study put forward a combination model to evaluate the computer network security. The combination model used particle swarm optimization (PSO) to optimize the parameters of BP neural network, speed up the BP neural network's convergence speed, and enhanced its global optimization ability, which effectively improved the accuracy of the evaluation model. Simulation results showed that compared with traditional BP neural network model, the combined model's learning ability was faster and global search ability was stronger, which effectively improved the evaluation accuracy of computer network security.

Keywords: Neural Network, Application, Computer Network Security Evaluation, PSO

Received: 10 March 2019, Revised 11 June 2019, Accepted 18 June 2019

DOI: $10.6025 / \mathrm{jic} / 2019 / 10 / 3 / 97-101$

(C) 2019 DLINE. All Rights Reserved

\section{Introduction}

With the gradual development of computer technology, people's living and working were enjoying great convenience from the advanced technology. At the same time, we also should clearly recognize that hackers can use loopholes, virus to invade computer system. Computer network security is facing unprecedented challenges. Neural network is widely used in network security technology evaluation. It is through the construction of artificial intelligence algorithms, and make the computer network security assessment more objective, higher accuracy. This article discussed the application of neural network in the computer network security evaluation and its related problems, and it could help the work of computer network security evaluation play a certain reference function in the future. Development of network technology has brought convenience to people's life, as well as the viruses, trojans and other destructive procedures attacking network. Computer network security is more and more been challenged [1]. It need accurately and scientifically evaluate the risk faced by the network, and to effectively

Journal of Intelligent Computing Volume 10 Number 3 September 2019 
guard against the risk, reduce the loss caused by computer network security problems [2].

Computer network security were under the influence of many factors, such as: intruding, vulnerabilities, viruses, related to each other and all kinds of factors. There were factors and the evaluation results between the complex nonlinear. Traditional evaluation methods, such as analytic hierarchy process, fault tree analysis, gray model for computer network security evaluation operation is complicated and difficult to accurately describe the nonlinear relationship, and quite low the evaluation accuracy [3]. Expert system is mainly associated with expert knowledge richness, evaluation result is subjective, instead of objective, without scientific, it is not suitable for complex computer network security evaluation. In recent years, neural network technology developed rapidly, and has self-learning ability, self-organization and strong adaptive capacity of artificial intelligence algorithms, simple implementation, stronger robustness. Artificial neural network model can effectively overcome the defect of traditional statistical model, through adjusting the weights of connections between neurons, the computer network security between the various properties of nonlinear capture, so as to realize the accurate evaluation on the computer network security, very suitable for the computer network security evaluation [4]. BP neural network (BPNN) is the most mature, and most widely used neural network, but as a result, the BP neural network is based on the gradient descent algorithm, there is a slow training speed and easy to fall into a tiny. The global search ability also has some shortcomings [5], The scope of affect and application, so how to improve the precision in the evaluation of neural network in the computer network security has become a focus in the field of computer network security research.

Particle swarm optimization (PSO) is a new developed bionic intelligent for searching algorithm simulation birds flying, with less number of individuals, simple calculation, and the advantages of global optimization performance is promising, and could carry on the optimization of neural network parameters [6]. According to the characteristics of the computer network security evaluation, combined with BP neural network nonlinear evaluation ability, this paper proposes PSO to optimize the BP neural network model of computer network security evaluation. The simulation proves that the model has a faster convergence speed, higher accuracy of evaluation.

\section{Principle of Computer Network Security Evaluation}

Computer network security evaluation principle is under the guidance of the evaluation standard. First of all, this study determines the evaluation content and scope, along with the current condition of network, and analyzes the security situation, the network vulnerabilities, then evaluates the relevant evaluation method, finally concludes the network security. The computer network security evaluation mathematical model is as below:

$$
\text { Network security level }=f\left(X_{1}, X_{2}, \ldots, X_{i}, \ldots X_{m}\right)
$$

With in this formula, $X_{i}$ stands for the computer network security evaluation factors, $f$ stands for the computer network security evaluation model.

From the computer network security evaluation model, it is crucial to choose the computer network security evaluation factor and network assessment model. Computer network security has the characteristics of uncertainty and nonlinear, so this article uses the BP neural network of nonlinear approximation ability as network evaluation model, using expert choice of computer network security factors, and carries on the scale, and determines the weights of the evaluation results, to improve the computer network security evaluation accuracy.

Computer network itself is a very complicated system, and factors which influence the safety of complexity and diversity, in order to effectively evaluate its security, and establish a perfect system of safety evaluation. The establishment of the computer network security evaluation system should follow the below principle: (1) Accuracy, to ensure that all evaluation indexes must be able to effectively reflect the real evaluation stage, and the network security technology; (2) Independence. In the selection of evaluation index, try to avoid a repeat choice; And try to reduce the correlation degree between various indicators, accurately and objectively reflect the network security situation; (3) Completeness, In the work, the selection of evaluation index shall to be thoughtful, appropriate choice, to ensure that the selected indicators, and for all the basic characteristics of network security are reported. It will make the selection of indicators be reliable, and ensure the accuracy of the results; (4) Briefness. Although the choice of evaluation index is to ensure its completeness, it also need to consider the actual workload and work efficiency in the evaluation index to select representative in ensure reliable results at the same time, reduced the workload.

98 Journal of Intelligent Computing Volume 10 Number 3 September 2019




\section{PSO-BPNN Model}

\subsection{Computer Network Security Evaluation Index Selection}

Computer network is a complex system, there are more factors effects the safety. In order to accurately evaluate their level of security, first of all, it shall establish a scientific and perfect computer network security evaluation index system. This article illustrates the management of the computer network system security, physical security and logical security. On the selected basis by experts of computer network security evaluation index, specific as shown in figure 1, and the expert system could consider computer network security evaluation index scores, and determine the weight.

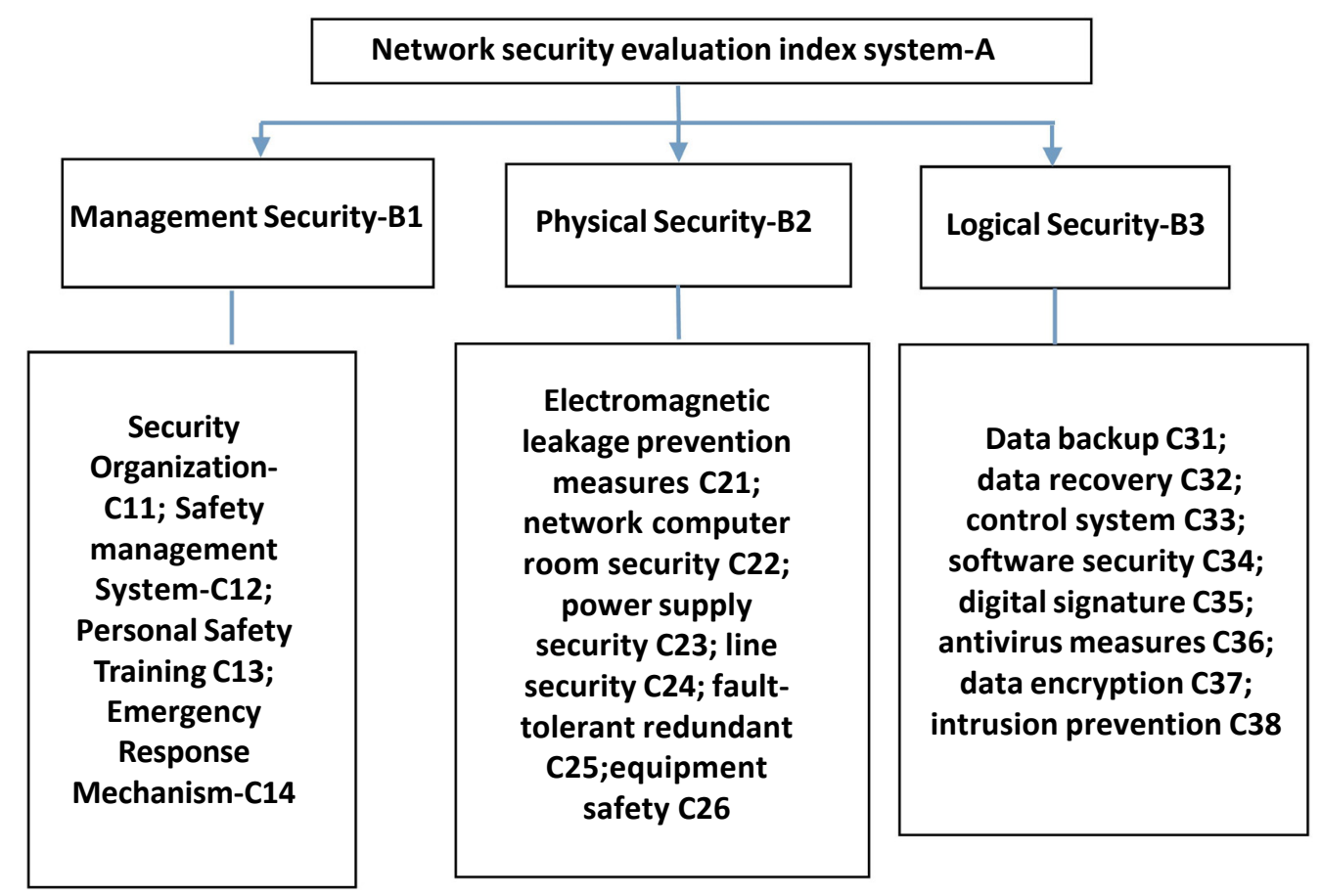

Figure 1. Computer network security evaluation index system

\subsection{Computer Network Safety Indexes of Normalized Processing}

Figure 1 building indicators reflects different angles for a computer network security, because a dimension of each index is different, and cannot be directly compared, in order to make the comparable between each index and accelerates the convergence speed of neural networks, this paper deals with the indexes normalization processing:

(1) Qualitative indicators: for the expert scoring method is used to determine its data, at the same time, it also normalized process the index.

(2) For the quantitative indexes: use the following formula for normalization processing.

$$
\text { Positive indicators: } \mathrm{x}_{i} \cdot=\left(\mathrm{x}_{i}-\mathrm{x}_{\text {imin }}\right) /\left(\mathrm{x}_{\text {imax }}-\mathrm{x}_{\text {imin }}\right)
$$

$\mathrm{x}_{i}{ }^{c}$ is for indicators for $\mathrm{x}_{i}$ normalized standard; $\mathrm{x}_{\text {imin }}, \mathrm{X}_{\text {imax }}$ respectively the minimum and maximum of the ${ }_{i}$ as an indicators.

\subsection{Computer Network Security Level Setting}

According to index of the comprehensive weight, it could be better to undertake safety evaluation of the computer network, compared with the related research, the computer network security level is divided into definitely security (A), the basic security unsafe (B), Not safe(C), safe been highly challenged (D), the level of security always set up for 1 point, the corresponding security level timely corresponding points are shown in table 1. 


\begin{tabular}{|l|l|l|l|l|}
\hline Classification & A & B & C & D \\
\hline Score & $1 \sim 0.85$ & $0.85 \sim 0.7$ & $0.7 \sim 0.6$ & $0.6 \sim 0$ \\
\hline
\end{tabular}

Table 1. Computer network security level

\subsection{The BP Neural Network Algorithm}

BP neural network is a multilayer feed forward network according to the error backward propagation, is one of the most widely used neural network model [7]. It uses gradient descent algorithm, by error back propagation network weights and threshold of constant adjustment, which is between the expectations of the neural network output and the actual output error sum of squares of the smallest. BP neural network has strong nonlinear approximation ability, method is simple, and easy in implementation, but easily trapped in local minima, which is difficult to guarantee the convergence to the global minimum point, global search ability is not strong. In addition, the BP neural network is based on the gradient descent algorithm of back propagation, the slow convergence speed and learning effect is difficult to satisfactory. In order to overcome the limitations of inherent in the BP neural network, this paper, by using PSO algorithm of BP neural network is optimized, and optimize the steps as follows:

1) The structure of the BP neural network, transfer function and target vector is initialized.

2) Set the size and parameters of particle swarm dimension, the number of iterations, the momentum coefficient, the initial position and velocity of particles.

3) Using the training set for acute trained BP neural network, and according to the type (5) to evaluate the fitness value of each particle.

4) Take the current value of each particle and best historical comparison, only if the current value is better than the best historical value, then save the current value of the particles for the history of the individual best value; Compare the current value of the particle swarm and its historical value, best better if its current value, is to save the current value of the history of the global best value.

5) The calculation of inertia weight.

6) The position and velocity of each particle is updated, record each particle and particle swarm fitness value error of system.

7) Fit value judgment system error, if error reached the set limit value or more than the maximum allowed number of iterations, then end the training. At this time the history of the particles to the global best position is the BP neural network weights and the optimal threshold.

\subsection{The Computer Network Security Evaluation Model}

This paper apples the PSO - BPNNPSO - BPNN model for computer network security evaluation, firstly constructs the evaluation index system of computer network security, then use particle swarm algorithm to optimize the BP neural network, have the best weights of BP neural network and optimal threshold, finally using the optimized BP neural network model for computer network security evaluation.

\section{The Simulation Research}

\subsection{The BP Neural Network Training}

This study applied the computer network security evaluation data set 45 groups before the data of BP neural network and PSO - BPNNPSO - the training BPNN model, due to the limitation of space, this article does not list the train diagram. From the training process, the traditional BP neural network convergence speed is slow, at the end of the 417th step training, error is 0.000984 , and the PSO - BPNNPSO - BPNN model after 312 iterations the error accuracy reached 0. 000761, than the traditional BP neural network model of the error of high precision, thus illustrates the slow convergence speed of traditional BP neural network, easily into the local minimum value, the overall optimization ability is not strong, greatly affect the evaluation precision of the model. And PSO - BPNNPSO - BPNN model due to the use of PSO algorithm for BP Ly effectively optimized via the network, not only makes the whole optimization ability get greatly improve and network training speed, and improve the training error precision effectively. 


\subsection{Results and Analysis}

When after learning of neural network to the expected accuracy, save the web, and then tested using the test set. The results show that the traditional BP neural network to 47 evaluation results of the computer network security data is wrong, the security level of sample data for class $\mathrm{C}$, which is unsafe, but its evaluation results are unsafe. Using PSO to optimize the BP neural network evaluation accuracy reached $100 \%$, according to root mean square error in the two models, the root mean square error is 0.067 , the traditional neural network is far greater than PS0 - root mean square error of BPNN model, the root mean square error brother only 0.023 , so as to illustrate the use of PS0 algorithm of BP neural network optimization is a success. Not only speed up the convergence speed of the network and model to improve the evaluation precision greatly.

\section{Conclusion}

In this paper, using the PSO algorithm and BP neural network was performed effectively, which is using the PSO algorithm to optimize the BP neural network. Make full use of the strong nonlinear function of BP neural network through close to capacity, using BP neural network for computer network security evaluation, considering that the BP neural network has the slow convergence speed into the bureau minute, no strong global search ability of the limits. Using PSO algorithm of BP neural network has carried on the effective optimization threshold, so as to obtain the weights of BP neural network is the best and most threshold, the optimization of the parameters of BP neural network got fundamental, effectively improve the accuracy of the computer network evaluation. Simulation results show that after the PSO algorithm. It need to optimize the BP neural network model of global search ability, evaluation precision, convergence rate significantly higher than the traditional BP neural network, illustrates the use of PSO algorithm. The optimization of BP neural network is successfully for future network evaluation providing a new attempt.

\section{References}

[1] Apostolopoulos, George., Gudrin, Roth., Kamat, Sanjay., Satish, K., Tripathi. (1998). Quality of service based routing: A performance perspective. 15-27.

[2] TANG, Chen-hua., YAO, Shui-ping., CUI, Zhong-jie. (2002). A network security policy model and its realization mechanism. Beilin:Springer-Verlag, 2002. 663-871.

[3] GUO, Li-juan. (2012). Neural network in the application of computer network security evaluation. Value Engineering.

[4] Brown, Bill., Cutts, Andrew., McGrath, Dennis. (2003). Simulation of cyber attacks with applications in homeland defense training. San Jose:SPIE Press, 63-70.

[5] MAO, Zhi-yong. (2008). Evaluation on BP neural network in the application of computer network security, Journal of Information Technology, 2008 (6).

[6] Michael, Liljenstam., Jason, Liu., David, Nicol. (2005). RINSE: The real-time immersive network simulation environment for network security exercises. San Diego, CA: Simulation Councils, 2005. 118-127.

[7] WU, Ren-jie. (2011). Security evaluation on neural network in the application of computer network. The Computer Simulation, $2011(11)$.

[8] Williams, L., Lippmann, R., Ingols, K. W. (2008). Garnet: A graphical attack graph and reachability network evaluation tool.

[9] Jin, J., Xu, H., LI B-C. (2011). Multicast scheduling with cooperation and network coding in cognitive radio networks. San Diego, California, USA, 2011. 1-9. 